\title{
Serum protein concentrations, including acute phase proteins, in calves with hepatogenous photosensitization
}

[Teores séricos de proteínas, inclusive proteinas de fase aguda, em bovinos com fotossensibilização hepatógena]

\author{
J.J. Fagliari ${ }^{1}$, M. Passipieri ${ }^{2}$, H.T. Okuda ${ }^{2}$, S.L. Silva ${ }^{3}$, P.C. Silva ${ }^{1}$ \\ ${ }^{1}$ Faculdade de Ciências Agrárias e Veterinárias - UNESP \\ Via de Acesso Prof. Paulo Donato Castellane, s/n \\ 14884-900 - Jaboticabal, SP \\ ${ }^{2}$ Faculdade de Engenharia de Ilha Solteira - Ilha Solteira, SP \\ ${ }^{3}$ Universidade Federal de Tocantins - Araguaína, TO
}

\begin{abstract}
One hundred 6- to 12-month-old Nelore calves were allotted into control group (G1; 50 healthy calves) and photosensitization group $(\mathrm{G} 2 ; \mathrm{n}=50)$. Blood samples were collected 12 to 24 hours after the onset of dermatitis (M1), and 15 to 30 days after that (M2), at time of resolution of clinical signs. Serum protein electrophoresis was performed by means of sodium dodecyl sulphate-polyacrylamide gel electrophoresis. Eighteen serum proteins with molecular weights ranging from 16,000 to 189,000 daltons (Da) were identified in all calves. In M1 and M2 serum concentrations of proteins with molecular weights of 115,000Da (ceruloplasmin), 61,000Da ( $\alpha_{1}$-antitrypsin), 45,000Da (haptoglobin), and 40,000Da (acid glycoprotein) were significantly increased in calves. In conclusion, measurement of serum acute phase protein concentrations may be useful in monitoring the progression of bovine hepatogenous photosensitization, including guide probable alteration on therapeutic procedures.
\end{abstract}

Keywords: calf, hepatogenous photosensitization, acute phase protein

\section{RESUMO}

Foram examinados 100 bezerros da raça Nelore com 6 a 12 meses de idade, distribuidos em: grupo controle $(G 1 ; 50$ bezerros sadios) e grupo fotossensibilização $(G 2 ; n=50)$. As amostras de sangue foram coletadas 12 a 24 horas após o início da dermatite (M1) e 15 a 30 dias após (M2), época da cura das lesões cutâneas. $O$ proteinograma sérico foi obtido por eletroforese em gel de acrilamida. Em todos os bezerros foram identificadas 18 proteinas com pesos moleculares (PM) entre 16.000 a 189.000 dáltons (Da). Em M1 e M2, as concentrações séricas das proteínas de PM 115.000Da (ceruloplasmina), 61.000Da ( $\alpha_{1}$-antitripsina), 45.000Da (haptoglobina) e 40.000Da (glicoproteína ácida) foram significativamente maiores em bezerros com fotossensibilização hepatógena em comparação com aquelas dos animais do grupo-controle. A determinação dos teores séricos de proteínas de fase aguda pode ser útil no monitoramento da progressão da fotossensibilização hepatógena em bovinos, inclusive orientando possíveis alterações em procedimentos terapêuticos.

Palavras-chave: bezerro, fotossensibilização hepatógena, proteína de fase aguda

\section{INTRODUCTION}

Bovine hepatogenous photosensitization has been reported in Brazil since 1975 (Dobereiner et al., 1976). Hepatotoxic saponins (Meagher et al., 1996; Cruz et al., 2000) and/or sporidesmin, mycotoxin from Pithomyces chartarum spores (Dobereiner et al., 1976; Fagliari et al., 1993ab; Fioravanti, 1999), have been incriminated as underlying cause of the disease in cattle grazing Brachiaria decumbens pastures. The disease causes substantial losses in animal production in several countries, including Brazil, where $B$. decumbens is a major forage provided to cattle.

Recebido em 7 de junho de 2006

Aceito em 17 de setembro de 2007

E-mail: gleidsongiordano@gmail.com 
As bovine hepatogenous photosensitization is an inflammatory condition, it is likely to induce changes in the serum concentration of acute phase proteins that could be identified by means of electrophoresis. If so, measuring changes in the acute phase proteins might be useful to detect and monitor progression of bovine photosensitization. Cellulose acetate (Fagliari et al., 1991) and agarose gel electrophoresis (Keay and Doxey, 1982) have been used to analyze plasma protein concentrations, but these techniques are limited, because they can identify only 5 to 7 groups of proteins. Sodium dodecyl sulphate-polyacrylamide gel electrophoresis (SDS-PAGE), on the other hand, can be used to indetify much more proteins, to separate proteins present in very low quantities, and can be performed on small serum or plasma samples (Gordon, 1975). The technique has been used to analyze plasma or serum protein concentrations (Coyne et al., 1990; Fagliari et al., 2003) but has not been previously used to determine changes in serum protein concentrations associated with hepatogenous photosensitization. The purpose of this study was to determine, by SDS-PAGE, the spectrum of serum protein alterations in bovine hepatogenous photosensitization, including acute phase proteins, and the rate at which these changes occur.

\section{MATERIALS AND METHODS}

One hundred 6- to 12-mounth-old Nelore calves were allotted into control group (G1; 50 healthy calves) and photosensitization group ( $\mathrm{G} 2 ; \mathrm{n}=50)$. Affect calves were kept or grazed on Brachiaria decumbens pastures. Diagnosis of hepatogenous photosensitization was based on history, clinical signs, and laboratorial findings (Towers and Stratton, 1978; Fagliari et al., 1993b). Blood samples were collected 12 to 24 hours after the onset of dermatitis (M1), and 15 to 30 days after that (M2), at time of resolution of skin lesions. Serum protein concentration was determined by SDS-PAGE (Weber and Osborn, 1969). Gels were stained for 5 minutes in $200 \mathrm{ml}$ of coomassie brilliant blue and destained in $7 \%$ acetic acid solution until the gel background was completely clear. Concentration of protein fractions was determined by use of computer-assisted videodensitometry ${ }^{1}$ Proteins were identified by use of reference markers ${ }^{2}$ with molecular weights of $29,000,45,000,66,000,97,400,116,000$, and 205,000 daltons (Da) and by comparison with electrophoretic mobility of purified albumin ${ }^{1}$,

${ }^{1}$ Shimadzu CS 9000, Shimadzu Corp. - Kyoto, Japan.

${ }^{2}$ Sigma Chemical Co. - St Louis, USA. transferrin ${ }^{1}$, haptoglobin ${ }^{1}$, ceruloplasmin ${ }^{1}, \operatorname{IgG}^{1}$, and $\alpha_{1}$-antitrypsin ${ }^{1}$.

Results of determinations were compiled, and results are presented as mean $\pm \mathrm{SD}$. Data were analyzed by use of repeated measure ANOVA. Means of interest were compared by use of Tukey test. A $\mathrm{P}$ value $<0.05$ was considered significant.

\section{RESULTS AND DISCUSSION}

Eighteen serum proteins with molecular weights ranging from 16,000 to $189,000 \mathrm{Da}$ were identified in all calves. Serum concentrations of proteins with molecular weights of $115,000 \mathrm{Da}$ (ceruloplasmin), $61,000 \mathrm{Da}\left(\alpha_{1}\right.$-antitrypsin), 45,000Da (haptoglobin), and 40,000Da (acid glycoprotein) were significantly increased in calves with hepatogenous photosensitization compared with concentrations in control calves (Table 1). These proteins have previously been identified as acute phase proteins (Gruys et al., 1994). Acute phase proteins are synthesized by the liver in response to inflammatory cytokines, particularly interleukin-6 (Heindrich et al., 1990).

Twelve to 24 hours after onset of dermatitis (M1), the protein with the highest percentage increase $(377.3 \pm 43.6 \%)$ in concentration was haptoglobin, compared with control group values (Table 2). At this time, percentage increase for ceruloplasmin, antitrypsin, and acid glycoprotein were $309.1 \pm 40.7 \%, \quad 251.7 \pm 23.8 \%$, and $146.2 \pm 22.5 \%$, respectively. Fifteen to 30 days after the onset of dermatitis (M2), at time of resolution of skin lesions, the protein with the highest percentage increase $(122.7 \pm 9.8 \%)$ in concentration was again haptoglobin, compared with control group values. At this time, percentage increase for ceruloplasmin, $\alpha_{1}$-antitrypsin, and acid glycoprotein were $51.4 \pm 8.9 \%, \quad 42.9 \pm 7.5 \%, \quad$ and $\quad 89.2 \pm 7.4 \%$, respectively, significantly lower than M1 values. Although acute phase protein concentrations in calves with photosensitization obtained in M1 have been lower than M2 (Table 1), these values were significantly higher than those verified in control calves, suggesting some persistent active lesion, probably in the liver, as showed in literature (Alessi et al., 1994; Fioravanti, 1999; Cruz et al., 2000). Even though acute phase proteins were produced in affected liver by stimulation of underlying cause of photosensitization, percentages increase were similar to those showed in calves with experimentally induced pneumonic pasteurellosis (Fagliari et al., 2003). 
Table 1. Serum protein concentrations (means \pm SD), determined by means of sodium dodecyl sulphatepolyacrylamide gel electrophoresis, in healthy calves (group 1) and in calves with hepatogenous photosensitization (group 2) obtained 12-24 hs after the onset of dermatitis (M1) and, 15-30 days after that (M2), at time of resolution of clinical signs

\begin{tabular}{|c|c|c|}
\hline Protein and group $(\mathrm{G})$ & M1 & M2 \\
\hline \multicolumn{3}{|c|}{ Total serum protein $(\mathrm{g} / \mathrm{dl})$} \\
\hline G1 & $6.43 \pm 0.52$ & $6.51 \pm 0.60$ \\
\hline $\mathrm{G} 2$ & $6.71 \pm 0.66$ & $6.60 \pm 0.57$ \\
\hline \multicolumn{3}{|c|}{ No identified $(\mathrm{MW}, 189,000 ; \mathrm{mg} / \mathrm{dl})$} \\
\hline G1 & $10.7 \pm 2.4$ & $11.4 \pm 2.9$ \\
\hline G2 & $11.9 \pm 3.0$ & $12.6 \pm 3.2$ \\
\hline \multicolumn{3}{|c|}{$\begin{array}{l}\text { Immunoglobulin A (MW, } 168,000 ; \mathrm{mg} / \mathrm{dl}) \\
\end{array}$} \\
\hline G1 & $258.3 \pm 15.1$ & $265.4 \pm 13.9$ \\
\hline G2 & $249.0 \pm 18.7$ & $256.1 \pm 16.7$ \\
\hline \multicolumn{3}{|c|}{ No identified $^{\ddagger}(\mathrm{MW}, 145,000 ; \mathrm{mg} / \mathrm{dl})$} \\
\hline G1 & $192.4 \pm 20.6$ & $186.3 \pm 21.6$ \\
\hline $\mathrm{G} 2$ & $209.6 \pm 18.3$ & $198.7 \pm 23.1$ \\
\hline \multicolumn{3}{|c|}{ Ceruloplasmin (MW, 115,$000 ; \mathrm{mg} / \mathrm{dl})$} \\
\hline G1 & $12.0 \pm 4.1 *$ & $13.4 \pm 2.7 *$ \\
\hline $\mathrm{G} 2$ & $49.1 \pm 6.2 \mathrm{a}$ & $20.3 \pm 3.1 \mathrm{~b}$ \\
\hline \multicolumn{3}{|c|}{ No identified $^{\ddagger}(\mathrm{MW}, 103,000 ; \mathrm{mg} / \mathrm{dl})$} \\
\hline G1 & $36.8 \pm 4.1$ & $35.0 \pm 3.5$ \\
\hline $\mathrm{G} 2$ & $34.3 \pm 2.9$ & $36.8 \pm 4.0$ \\
\hline \multicolumn{3}{|c|}{ Phosphorylase A (MW, 91,000; mg/dl) } \\
\hline G1 & $45.7 \pm 3.8$ & $44.0 \pm 3.2$ \\
\hline G2 & $47.0 \pm 4.2$ & $48.1 \pm 5.0$ \\
\hline \multicolumn{3}{|c|}{ Transferrin (MW, 80,000; mg/dl) } \\
\hline G1 & $264.9 \pm 19.5$ & $271.1 \pm 21.7$ \\
\hline G2 & $252.7 \pm 20.9$ & $264.3 \pm 25.3$ \\
\hline \multicolumn{3}{|c|}{ Albumin (MW, 66.000; g/dl) } \\
\hline G1 & $3.31 \pm 0.26$ & $3.41 \pm 0.29$ \\
\hline $\mathrm{G} 2$ & $3.18 \pm 0.40$ & $3.27 \pm 0.33$ \\
\hline \multicolumn{3}{|c|}{ IgG high-chain $(\mathrm{MW}, 62,000 ; \mathrm{mg} / \mathrm{dl})$} \\
\hline G1 & $564.4 \pm 50.6$ & $572.3 \pm 46.7$ \\
\hline $\mathrm{G} 2$ & $581.7 \pm 55.1$ & $570.0 \pm 51.0$ \\
\hline \multicolumn{3}{|c|}{$\overline{\alpha_{1} \text {-antitrypsin }(\mathrm{MW}, 59,000 ; \mathrm{mg} / \mathrm{dl})}$} \\
\hline G1 & $23.4 \pm 3.6^{*}$ & $21.4 \pm 2.2 *$ \\
\hline G2 & $82.3 \pm 14.4 \mathrm{a}$ & $30.6 \pm 4.3 \mathrm{~b}$ \\
\hline \multicolumn{3}{|c|}{ No identified ${ }^{\ddagger}(\mathrm{MW}, 53,000 ; \mathrm{mg} / \mathrm{dl})$} \\
\hline G1 & $18.6 \pm 3.1$ & $17.6 \pm 3.4$ \\
\hline G2 & $21.4 \pm 4.0$ & $20.4 \pm 3.9$ \\
\hline \multicolumn{3}{|c|}{$\overline{\text { Haptoglobin (MW, 45,000; mg/dl) }}$} \\
\hline G1 & $32.3 \pm 4.0 *$ & $36.5 \pm 3.7 *$ \\
\hline G2 & $154.2 \pm 26.2 \mathrm{a}$ & $81.3 \pm 10.4 \mathrm{~b}$ \\
\hline \multicolumn{3}{|c|}{ Acid glycoprotein (MW, 40,000; mg/dl) } \\
\hline G1 & $14.5 \pm 2.8^{*}$ & $11.2 \pm 1.9^{*}$ \\
\hline G2 & $35.7 \pm 5.8 \mathrm{a}$ & $21.2 \pm 2.8 \mathrm{~b}$ \\
\hline \multicolumn{3}{|c|}{ No identified $^{\ddagger}(\mathrm{MW}, 38,000 ; \mathrm{mg} / \mathrm{dl})$} \\
\hline G1 & $28.6 \pm 3.9$ & $26.0 \pm 3.5$ \\
\hline $\mathrm{G} 2$ & $30.5 \pm 3.4$ & $31.1 \pm 4.0$ \\
\hline \multicolumn{3}{|c|}{ No identified ${ }^{\ddagger}(\mathrm{MW}, 36,000 ; \mathrm{mg} / \mathrm{dl})$} \\
\hline G1 & $141.7 \pm 12.6$ & $152.1 \pm 15.0$ \\
\hline G2 & $150.8 \pm 14.1$ & $161.4 \pm 18.3$ \\
\hline \multicolumn{3}{|c|}{$\overline{\mathrm{IgG}}$ light-chain (MW, 32,000; mg/dl) } \\
\hline G1 & $822.7 \pm 53.9$ & $836.1 \pm 60.3$ \\
\hline G2 & $880.1 \pm 76.3$ & $902.0 \pm 82.4$ \\
\hline \multicolumn{3}{|c|}{ No identified $^{\ddagger}(\mathrm{MW}, 26,000 ; \mathrm{mg} / \mathrm{dl})$} \\
\hline G1 & $371.6 \pm 28.9$ & $360.8 \pm 31.9$ \\
\hline G2 & $392.3 \pm 32.6$ & $381.7 \pm 40.6$ \\
\hline \multicolumn{3}{|c|}{ Hemoglobin (MW, 16,$000 ; \mathrm{mg} / \mathrm{dl})$} \\
\hline G1 & $30.6 \pm 5.1$ & $28.7 \pm 4.5$ \\
\hline $\mathrm{G} 2$ & $36.0 \pm 6.4$ & $33.2 \pm 5.0$ \\
\hline
\end{tabular}

*Significantly $(\mathrm{P}<0.05)$ different from values for calves with hepatogenous photosensitization.

In each row, values with different letters were significantly $(\mathrm{P}<0.05)$ different from each other.

$\mathrm{MW}=$ molecular weight.

${ }^{\dagger}$ No identified proteins because there were no purified proteins to compare (like ceruloplasmin, haptoglobin, transferring) 
Table 2. Serum acute phase protein concentrations (means \pm SD) and percentage increase in serum acute phase protein concentrations, compared with healthy calves concentrations, in calves with hepatogenous photosensitization obtained 12-24 hs after the onset of dermatitis (M1) and, 15-30 days after that (M2), at time of resolution of clinical signs

\begin{tabular}{|c|c|c|}
\hline Protein & M1 & M2 \\
\hline \multirow[t]{3}{*}{ Ceruloplasmin } & G1: $12.0 \mathrm{mg} / \mathrm{dl}$ & G1: $13.4 \mathrm{mg} / \mathrm{dl}$ \\
\hline & $\mathrm{G} 2: 49.1 \mathrm{mg} / \mathrm{dl}$ & $\mathrm{G} 2: 20.3 \mathrm{mg} / \mathrm{dl}$ \\
\hline & $309.1 \pm 40.7 \%$ & $51.4 \pm 8.9 \%$ \\
\hline \multirow[t]{3}{*}{$\alpha_{1}$-antitrypsin } & $\mathrm{G} 1: 23.4 \mathrm{mg} / \mathrm{dl}$ & $\mathrm{G} 1: 21.4 \mathrm{mg} / \mathrm{dl}$ \\
\hline & $\mathrm{G} 2: 82.3 \mathrm{mg} / \mathrm{dl}$ & $\mathrm{G} 2: 30.6 \mathrm{mg} / \mathrm{dl}$ \\
\hline & $251.7 \pm 23.8 \%$ & $42.9 \pm 7.5 \%$ \\
\hline \multirow[t]{2}{*}{ Haptoglobin } & $\mathrm{G} 1: 32.3 \mathrm{mg} / \mathrm{dl}$ & $\mathrm{G} 1: 36.5 \mathrm{mg} / \mathrm{dl}$ \\
\hline & $\begin{array}{l}\mathrm{G} 2: 154.2 \mathrm{mg} / \mathrm{dl} \\
377.3 \pm 43.6 \%\end{array}$ & $\begin{array}{l}\mathrm{G} 2: 81.3 \mathrm{mg} / \mathrm{dl} \\
122.7 \pm 9.8 \%\end{array}$ \\
\hline \multirow[t]{2}{*}{ Acid glycoprotein } & $\mathrm{G} 1: 14.5 \mathrm{mg} / \mathrm{dl}$ & $\mathrm{G} 1: 11.2 \mathrm{mg} / \mathrm{dl}$ \\
\hline & $\begin{array}{r}\mathrm{G} 2: 35.7 \mathrm{mg} / \mathrm{dl} \\
146.2 \pm 22.5 \%\end{array}$ & $\begin{array}{r}\mathrm{G} 2: 21.2 \mathrm{mg} / \mathrm{dl} \\
\qquad 89.2 \pm 7.4 \%\end{array}$ \\
\hline
\end{tabular}

\section{CONCLUSION}

Measurement of serum acute phase protein concentrations may be useful in monitoring the progression of bovine hepatogenous photosensitization, including guide probable alteration on therapeutic procedures.

\section{REFERENCES}

ALESSI, A.C.; FAGLIARI, J.J.; BECHARA, G.H. Intoxicação natural de bovinos pela micotoxina esporidesmina: Lesões hepáticas. Arq. Bras. Med. Vet. Zootec., v.45, p.471-478, 1994

COYNE, C.P.; CARLSON, G.P.; SPENSLEY, M.S. et al. Preliminary investigation of alterations in blood viscosity, cellular composition and electrophoresis plasma protein fraction profile after competitive racing activitie in Thoroughbred horses. Am. J. Vet. Res., v.51, p.1956-1963, 1990.

CRUZ, C.; DRIEMEIER, D.; PIRES, V.S. et al. Isolation of steroidal sapogenins implicated in experimentally induced cholangiopathy of sheep grazing Brachiaria decumbens in Brazil. Vet. Human Toxicol., v.42, p.142-145, 2000.

DOBEREINER, J.; TOKARNIA, C.H.; MONTEIRO, M.C.C. Intoxicação de bovinos e ovinos em pastos de Brachiaria decumbens contaminadas por Pithomyces chartarum. Pesq. Agropec. Bras., série veterinária, v.11, p.87-94, 1976.

FAGLIARI, J.J.; OKUDA, H.T.; PASSIPIERI, M. et al. Serum protein levels of Guzera cattle in different ages. Arq. Bras. Med. Vet. Zootec., v.43, p.39-60, 1991.

FAGLIARI, J.J.; OKUDA, H.T.; KUCHEMBUCK, M.R.G. et al. Intoxicação natural de bovinos pela micotoxina esporidesmina. I. Aspectos epidemiológicos. Arq. Bras. Med. Vet. Zootec., v.45, p.263-274, 1993a.
FAGLIARI, J.J.; OKUDA, H.T.; KUCHEMBUCK, M.R.G. et al. Intoxicação natural de bovinos pela micotoxina esporidesmina. II. Aspectos clínicos. Arq. Bras. Med. Vet. Zootec., v.45, p.275-282, 1993 b.

FAGLIARI, J.J.; WEISS, D.J.; McCLENAHAN, D. et al. Serum protein concentrations in calves with experimentally induced pneumonic pasteurellosis. Arq. Bras. Med. Vet. Zootec., v.55, p.383-387, 2003.

FIORAVANTI, M.C.S. Incidência, avaliações clínica, laboratorial e anatomopatológica da intoxicação subclínica por esporidesmina em bovinos. 1999. 256f. Tese (Doutorado) - Faculdade de Medicina Veterinária e Zootecnia, Universidade Estadual Paulista, Botucatu, SP.

GORDON, A.H. Electrophoresis of proteins in polyacrylamide and starch gels. New York: Elsevier Science Publishers, 1975. 213p.

GRUYS, E.; OBWOLO, M.J.; TOUSSAINT M.J.M. Diagnostic significance of the major acute phase proteins in veterinary clinical chemistry: A review. Vet. Bull., v.64, p.1009-1018, 1994.

HEINDRICH, P.C.; CASTELL, J.V.; ANDUS, T. Interleukin-6 and the acute phase response. Biochem. J., v.265, p.621-636, 1990.

KEAY, G.; DOXEY, D.L. Species characteristics of serum proteins demonstrated after agarose gel electrophoresis. Vet. Res. Commun., v.5, p.263-270, 1982.

MEAGHER, L.P.; WILKINS, A.L.; FAGLIARI, J.J. et al. Hepatogenous photosensitization of ruminants by Brachiaria decumbens and Panicum diclotomiflorum in the absence of sporidesmin: lithogenic saponins may be responsible. Vet. Human Toxicol., v.38, p.271-273, 1996.

TOWERS, N.R.; STRATTON. G.C. Serum gammaglutamyltransferase as measure of sporidesmin-induced liver damage in sheep. New Zeal. Vet. J., v.26, p.109-112, 1978.

WEBER, K.; OSBORN, M. The reliability of molecular weight determinations by dodecyl sulfate-polyacrylamide gel electrophoresis. J. Biol. Chem., v.214, p.4406-4412, 1969. 